\title{
Knowledge Integration to Support Networking for Laboratory Preparedness and Response to Emerging Pathogens
}

\author{
Shamir N. Mukhi ${ }^{1}$, Lai King Ng${ }^{1}$, Theodore I. Kuschak ${ }^{1}$ and May Chu ${ }^{2}$ \\ 1Public Health Agency of Canada \\ ${ }^{2}$ Centers for Disease Control and Prevention \\ ${ }^{1}$ Canada \\ 2United States of America
}

\section{Introduction}

Laboratories play a critical role in facilitating timely recognition of and response to public health threats. However, capabilities and capacities vary widely among laboratories around the world. The scientific community recognizes that: 1) no single laboratory or network can effectively cover all health hazard threats and 2) connecting laboratories through networks enables scientific communities to harness and contribute their expertise in response to public health threats, while adding value and enhancing opportunities to enrich their own work. However, a consolidated and accessible inventory of laboratories that would enable this to happen does not exist.

Public health laboratories serve the essential function of identifying etiologic agents of disease in an accurate and timely manner. However, the practicality and potential of these laboratories in the detection, monitoring, and reporting of threats over a wider geographic range is limited by unclear case definitions, inadequate laboratory capacity, and often, limited political will of local authorities to comply with International Health Regulations (IHR) 2005 (Baker and Fidler, 2006) to be prepared to respond to public health emergencies of international concern (PHEIC). Furthermore, some countries are not member states of World Health Organization (WHO) and therefore have no obligation to comply with IHR.

Global, regional, and national laboratory networks serve to alleviate these issues by streamlining the detection, monitoring, and reporting procedures for communicable diseases in order to effectively and significantly reduce the global or regional burden of disease. Laboratory networks are useful in establishing and maintaining standards such as molecular disease confirmation by providing member laboratories with standardized testing and reporting procedures, reagents, equipment, training, reference materials, quality control indicators, and technical support. Such collaboration between and among laboratories facilitated by networks allows for rapid and accurate provision of information regarding the magnitude of disease and the strains that are circulating in particular regions, leading to faster response and more effective control of the threat. Some example networks include the Global Polio Network (Hull et al, 1997) and the Global Measles and Rubella Network (Featherstone et al, 2003). 
Although significant scientific knowledge has been developed, tested and translated into successful public health interventions and leading to reduced infectious disease burden, the public remains vulnerable to epidemics and pandemics. Emerging infections such as HIV, SARS, avian influenza and the recent H1N1 pandemic are further exemplified by the emergence and global spread of multi-drug resistant pathogens, which threatens our ability to treat viral and bacterial infections in hospitals and in the community. Taken together these acts of nature have put enormous pressure on governments to act quickly to protect the public's health. The public have in turn, incurred high costs in terms of lives and implementation of countermeasures. These public health problems have resulted in society disturbance, economic loss and political expectations.

\section{Global laboratory directory}

The WHO revised the IHR in 2005. This revision updated the practice of reporting and monitoring emerging threats of international concern and calls for strengthening of core capacities, including the laboratories of its 194 member countries (WHO, 2008).

The implementation of IHR capacity plan is often an iterative process. The global scientific community recognizes that no single laboratory or country has the resources to control all health hazards at all times. In this chapter, we focus on infectious diseases, but these principles also apply to chemical and radio-nuclear threats.

Infectious diseases do not recognize geographic borders. Cross-border collaborations and information sharing are necessary for early mitigation of a PHEIC caused by infectious agents. Therefore, it is important that laboratories also tap into existing external resources while strengthening their own capacity at the same time. One such resource is the vibrant community of laboratory networks. In this age of rapid communication and creation of virtual communities of practice, connectivity and information accessibility becomes easier, yet loses the assurance of scientific scrutiny. Networks of laboratories that come together as a community of practice can provide reliable information and expertise. In Canada, we have established many different types of networks to serve different purposes. These include disease specific networks, public health networks, hospital networks and networks of networks. There are also many successful international and global networks that serve as invaluable resources to public health. However, locating specific laboratories or networks can be laborious using publically available search engines, especially since many are not visible on the web.

Over the last decade, we have witnessed significant change in the way scientific communities share experiences, exchange methods and ideas, expertise and resources through use of emerging Internet and satellite technologies. The ease of travel, access to ejournals, rapid Internet searches, daily outbreak updates and virtual meetings further create opportunities to meet and connect in ways never possible in the past. These electronic gateways are becoming more accessible to even those laboratories that have been the most remote and isolated. Scientists, laboratories, and networks have embraced these developments, but the remaining challenge is to capture and share this ever-expanding, vibrant, and science-based knowledge in a simple and intuitive manner. Some of the recent attempts to developing web based search engines are not truly designed for laboratories and, as such do not have an efficient means of filtering information relevant to laboratories. Even some of the popular websites have proven difficult to navigate. The connectivity to experts and access to information is important for global laboratory capacity building. It is 
important to sustain laboratory networks that could serve as irreplaceable repositories of experience and knowledge.

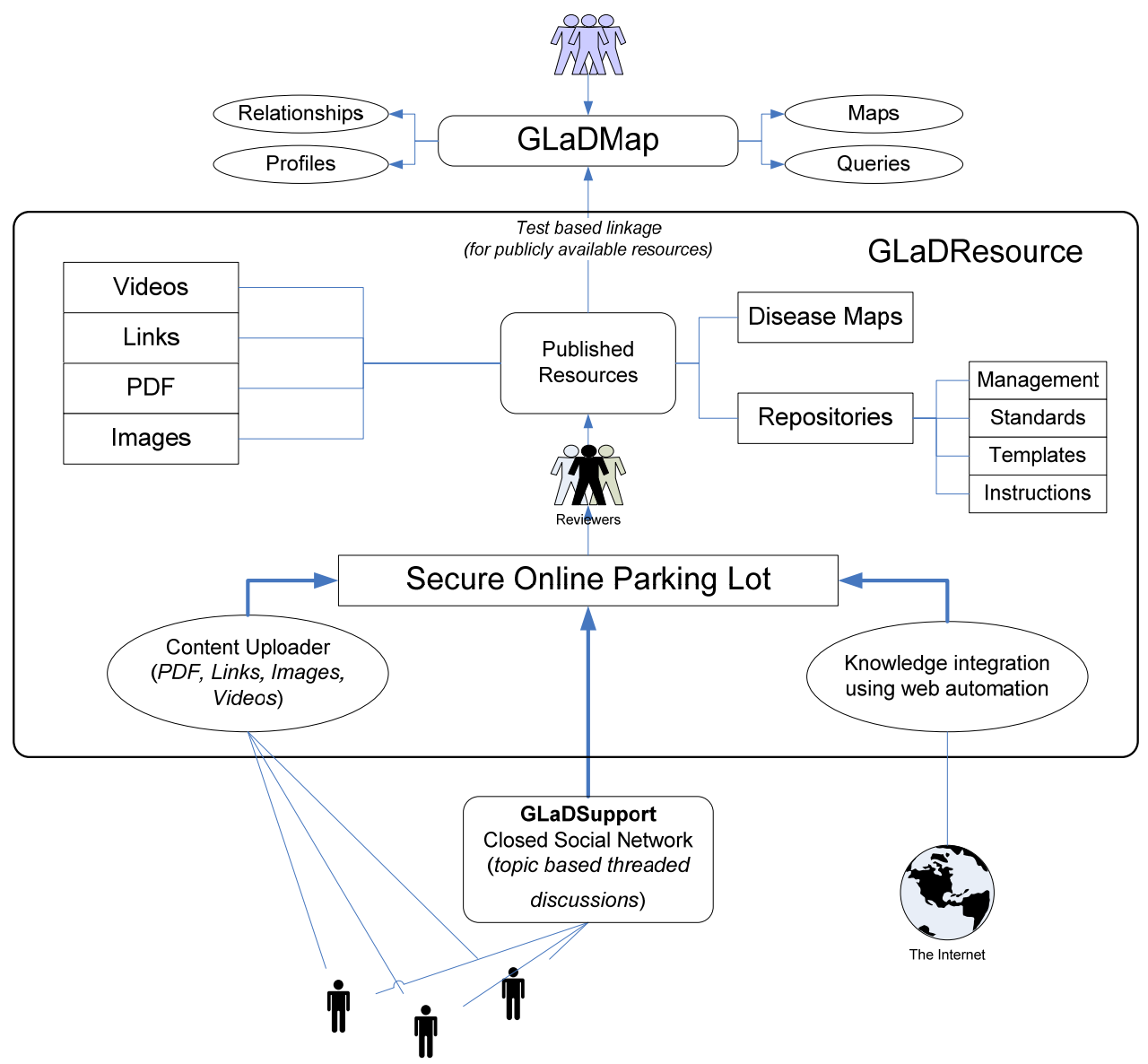

Fig. 1. Global Laboratory Directory Vision

The WHO, the National Microbiology Laboratory, Public Health Agency of Canada and Centers for Disease Control and Prevention, USA collaborated on developing the Global Laboratory Directory (GLaD) to map, connect, and support the laboratory networks and their members. GLaD is conceived as a support system to encourage laboratory networks to be part of a global community of peers. It is to connect laboratories among and between networks to leverage their capabilities and capacities in support of effective preparedness and in compliance with the IHR. GLaD, as depicted in Fig. 1, comprises of three main components: GLaDMap, GLaDResource and GLaDSupport.

It is easy to undervalue laboratories as assets for health protection and to question the need for investment into them and their existence. Moreover, the power of networking as a means of achieving public health goals is not always explored or harnessed. GLaD aims to support 
laboratory networks and advocate for their support and sustainability for the following reasons:

- Networks embody collaborative spirit and enthusiasm as players in the health sector

- Their collective work enriches scientific knowledge and range of expertise

- They contribute critical resources, capacity, and capability in the form of organization, technical experts, materials, information and innovation

- Scientific knowledge must be built through multi-disciplinary partnerships, and participation of many members and organizations is more comprehensive and timely than those derived from individual efforts

- Supporting networks is a practical and efficient way to capture and retrieve the technical and corporate knowledge, experience and resources for the public good

GLaD priorities and activities are aligned and complimentary to the United Nations Millenium Development Goals, with existing global health regulations and codes in animal, plant, and human sectors, and with global laboratory initiatives. The GLaD project adheres to the following core principles:

- Ensure equity for all participants

- Respect community rights and national sovereignty

- Listen and respond to the needs of the stakeholders

- Respect autonomy of existing networks - Add value to their core functions and activities

- Consult with wide-ranging experts and users to obtain a balanced view

- Recognize laboratory expertise wherever it resides

- Acknowledge participant contributions

- Operate with transparency and adhere to highest ethical practices

GLaD platform is developed using the established Canadian Network for Public Health Intelligence (CNPHI) technology framework (Mukhi et al, 2007), which is a comprehensive framework of applications and resources designed to fill critical gaps in Canada's national public health infrastructure. CNPHI's innovative Program/Information Technology approach supports data sharing and collaboration by integrating disparate data sources and facilitating intelligence generation and dissemination to enhance public health coordination and response activities for the direct benefit of local, regional, provincial/territorial and national stakeholders.

\subsection{GLaDMap}

\subsubsection{Overview}

The GLaDMap component provides an intuitive multi-faceted search engine with visual depiction of the interactive relationships of the networks and their member laboratories using a visualization tool that displays information provided by the network and by member laboratories themselves. GLaDMap enables users to: (1) find laboratories or networks that are dedicated to a specific objective or function, (2) connect with those located in geographical locations of interest, and (3) search for specific type(s) of services and, expertise/experts.

GLaDMap comprises of three fundamental hierarchical information units: 1) Laboratory, 2) Institution and 3) Network. A laboratory is defined as a place (room, building or facility) set apart for a group of scientists to conduct practical investigations in science (e.g. bacteriology, virology, toxicology, parasitology) or production of reagents for such 
investigations (animal facility, media preparation) and analysis and interpretation of results (e.g. bioinformatics, biostatics, mathematical modeling) from undertaking the investigations. A laboratory can be complex, multi-faceted or very simple, set up for basic sample collection as its sole function. A laboratory is the most basic information unit within the GLaDMap system. The following rules apply for including a laboratory on GLaD:

- Every laboratory named in a network is included in GLaDMap

- All national reference centres, including those not currently in laboratory networks, but which have the mandate from their respective state/national authority to provide a laboratory service to the public in a country

- $\quad$ Regional reference centres that have been nominated by peers or by official authorities to provide public health laboratory services to a specific region

- WHO Collaborating Centers that provide specific laboratory services

The following types of laboratories are to be excluded from GLaD:

- Laboratories in profit-driven institutions

- Laboratories that conduct research that generates intellectual properties that reduces global equity in access to laboratory services or diagnostic tests

- Laboratories or those institutions that market products of public health risks.

It is understood that the management structure and organization of institutes may differ so as to optimize the delivery of laboratory services to their external stakeholders, clients, or communities. Laboratories established within an institution may be set up based on specific syndromes (e.g. respiratory, diarrhoeal), methodology platforms (serology, DNA based or genotypic methods (e.g. PCR, DNA sequencing, culture or phenotypic methods), specific functions (research and development, technology transfer, surveillance, reference services, emergency preparedness and response), or a combination of approaches. GLaDMap does not place restrictions for participation on size, types, or management system of laboratories, but it is important to be able to easily locate the appropriate person responsible for further communications.

The second information unit within GLaDMap is an institution, which is defined as an organization established to provide public services. It has one or more laboratory units, which are the official workspaces of scientists and principal investigator(s) where they carry out their scientific work. An institution is a legal entity that has an official designation that gives them the mandate or authority to house the laboratory units. Any number of laboratory units may be co-located within the same institution. The following rules apply for including an institution on GLaD:

- Institutions that host laboratories that are member of networks included in GLaDMap

- Those that have national reference laboratories

- Institutions that have mandate from the state to provide reference services to the public

- Institutions that host WHO Collaborating Centres having a laboratory function Institutions hosting laboratories that provide data for national or global laboratory surveillance

- Institutions hosting laboratories that conduct research to answer specific public health questions, health hazard characterization, hazard identification, and hazard detection without profit.

All profit driven institutions that are not members of networks within GLaD should be excluded. 
The third information unit with GLaDMap system is a network, which is defined as an interconnected entity (usually championed by a "leader" or "manager") and linked by common interest (a community of practice). Members develop a working relationship for professional benefit and visibility. Often these networks are established to achieve common goals that are accomplished more easily together than by one or two entities on their own. These networks may have formal or informal organizational and administrative structures. The following rules apply for including a network on GLaD:

- Societies, consortiums, and committees that provide laboratory service to public without monetary profit.

- Laboratory network members that are representatives of their laboratories or institutions that are part of the public health system.

- Laboratory networks that do not endorse a specific commercial organization or institution

- Public health institutions or states that could benefit from the promotion of laboratory networks found in GLaDMap

- Laboratory networks of importance for improving preparedness in prevention, response, and control of public health emergencies of global concern.

- Laboratory networks that provide data required for public health functions and decision-making e.g. surveillance, patient diagnosis, reference services, research and development, transfer of laboratory technologies and human resource development.

The following types of networks must be excluded from GLaD:

- Professional societies based on contributions of individual members to advance the knowledge in science. Their members represent their own achievements and positions, and rarely those of their institution, organizations, laboratories or employers.

- Societies, consortiums or advisory committees (groups, bodies) that play an active role in a policy advisory capacity. These societies could be linked to GLaD, but not part of GLaDMap

- Societies or networks who could be players in public health, but represent the interests of private or commercial organizations

- Networks that play an active role in advocacy of specific subject matter that may be in conflict with government or government funded laboratory policies.

\subsubsection{Functionality}

The GLaDMap system includes three information units as mentioned earlier, including Laboratory, Institution and Network. Each of these units is represented on the system by a collection of data elements referred to as a profile. The laboratory profile form consists of the following sections that may be completed by the participating sites as available:

- Identity: Laboratory name, website information, and contact information including geographical information so that the laboratory can be mapped.

- Disciplines: Classification information, such as, bacteriology, chemistry, immunology, toxicology, et cetera.

- Functions: Main role and responsibilities of the laboratory. Some examples include, clinical diagnostics, surveillance, outbreak investigation, vaccine production, specimen banking, training, reference services, quality assurance and veterinary.

- Specimen: Environmental (such as, food, water, soil, air), Clinical, Toxicology, Animal, and information on number of containments units at each level for handling specimens. 
- Tests: Bacteriology, Immunology, Mycology, Parasitology, Toxicology, Virology, including information on estimates of number of specimens handled yearly and quality management system.

The institution profile form consists of the following sections that may be completed by the participating sites as available:

- Identity: Institution name, website information, and contact information including geographical information so that the institution can be mapped.

- Laboratories: List of member laboratories associated with the institution or network.

- Networks: List of networks that the institution is affiliated with.

The network profile form consists of the following sections that may be completed by the participating sites as available:

- Identity: Network name, website information, and contact information including geographical information so that the network can be mapped.

- History: Captures the purpose of network's establishment and any associated history including governance body, funding sources, terms of reference and founders.

- Activities: Descriptions that best describe the network activities including workshops and annual meetings.

- Disciplines: Classification information for member laboratories, such as, bacteriology, chemistry, immunology, toxicology, etc.

- Functions: Main role and responsibilities of the member laboratories. Some examples include, clinical diagnostics, surveillance, outbreak investigation, vaccine production, specimen banking, training, reference services, quality assurance and veterinary.

- Type: Local, national, international, global or regional.

- Laboratories: List of member laboratories associated with the institution.

The GLaDMap tool has been developed as an online web-based system to enable quick access to posted profiles and to provide the ability to seamlessly create or update new profiles while keeping the ownership of data in mind. In order to begin the data entry, GLaD has defined the persons expected to enter the information as follows (as illustrated in Fig. 2):

- The leader or manager (or a designate) of a network is the person to enter information for the network profile in GLaDMap

- The director of the institution is the official representative (or a designate) to enter the data for the institutional profile.

- The leader of the laboratory unit is the responsible person (or a designate) to enter data for the laboratory profile.

A laboratory must be affiliated with a network for it to exist within the GLaDMap system. This relationship defines the basis of the integrated search engine to define relationships between networks and their members. Optionally, a laboratory might be associated to an institution as intermediary organization between a network and laboratory. This hierarchical structure enables multi-dimensional relationships to co-exist between networks, institutions and laboratories. For example, one laboratory can be a member of multiple networks; an institution can have multiple laboratory members and be affiliated with multiple networks.

Fig. 3 is a functionality chart illustrating the data flow of the application including the searching functionality as well as the update/submit new profile functionality. Note that a laboratory may belong to a network directly without belonging to an institution. 
In summary, a user needs to follow the following steps upon visiting the home page:

- Select the profile: Network, Institution or Laboratory

- Enter the relevant information in each of the sections

- Submit the profile with associated email address for confirmation and follow-up if required

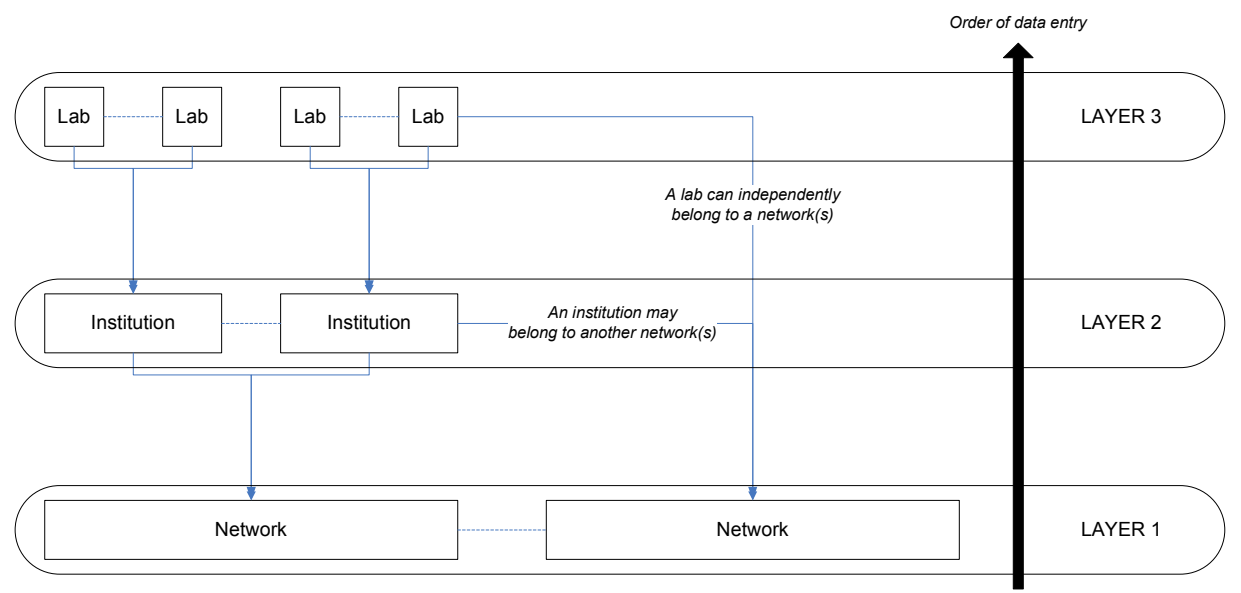

Fig. 2. GLaDMap: Illustration of three hierarchical data entry layers

Once the profiles have been entered, the information is automatically submitted into a restricted pending box within the online GLaDMap system. The profiles are then reviewed and approved by the GLaDMap Reviewer (who may consult with the data provider before approving the profile). Once approved, the profile immediately becomes publicly available for searching and the submitter is notified via automatically generated email.

The GLadMap system includes a mechanism to update existing profiles by the responsible individuals using a built-in email based one-time use access links. This mechanism alleviates the need for user account management. This is achieved by sending an automatically generated single use link in an email to the profile lead requesting an update. Fig. 4 illustrates steps required to submit/update each of the profiles using the GLaDMap system.

The search functionality provides an interactive mechanism for users to find laboratories using one of the four available search methods: Laboratory Name, Network, Geographical Location, and Tests performed. These methods may be combined to further filter the results. The system provides the results in one of three ways: Simple line list (which displays the results in a list highlighting some of the key data elements including name, primary contact, country and link to the detailed profile); color coded geographical point maps using integrated Google Maps; and proprietary interactive visualization tool called interactive relationship diagram (IRD), as illustrated in Fig. 5.

The IRD is a used to visualize multi-dimensional relationships between and among laboratories, institutions, networks, countries, and WHO regions. The tool allows user to visually interact with the relationships and analyze various patterns that can emanate from such interaction. 


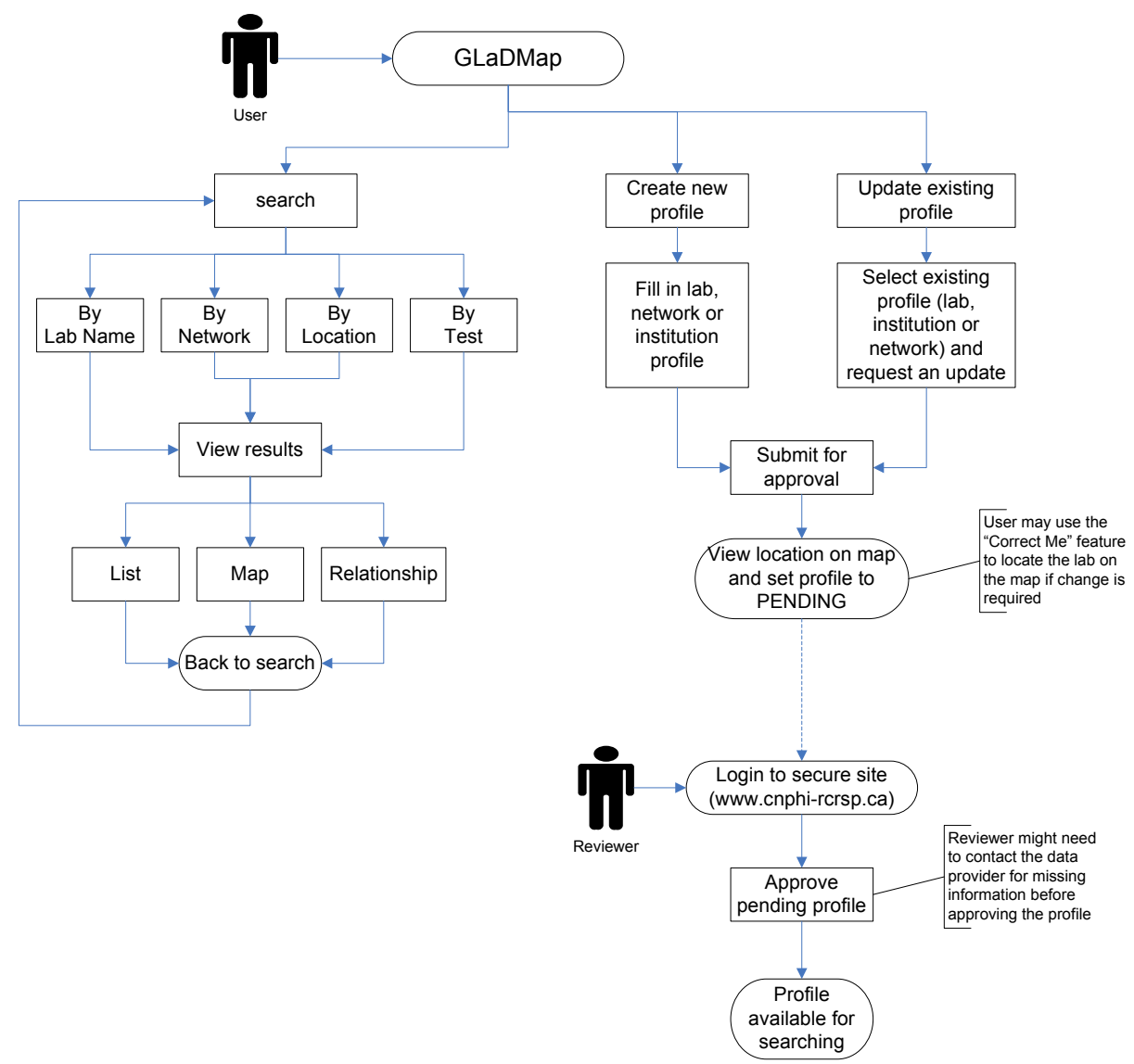

Fig. 3. GLaDMap: Application data flow for searching, creating and updating profiles

\subsubsection{Preliminary analysis}

GLaDMap currently contains 33 laboratory networks and over 1,000 laboratories. Analysis of network and laboratory profile completion has shown that gaps still exist in the completeness of the networks, and only $10 \%$ of laboratories have complete profiles at this time. Some preliminary analysis of search usage data has shown that the most commonly used search fields were network and test performed. This underscores the importance of network and profile completion, as it increases the likelihood of the search term appearing in the search results.

The next step is to perform in-depth analysis of the data that has been collected using the GLaDMap tool to identify missing data, most commonly completed fields, fully completed networks, and search usage. Such analysis will aid in understanding the use of the system and assist in planning the next steps. 


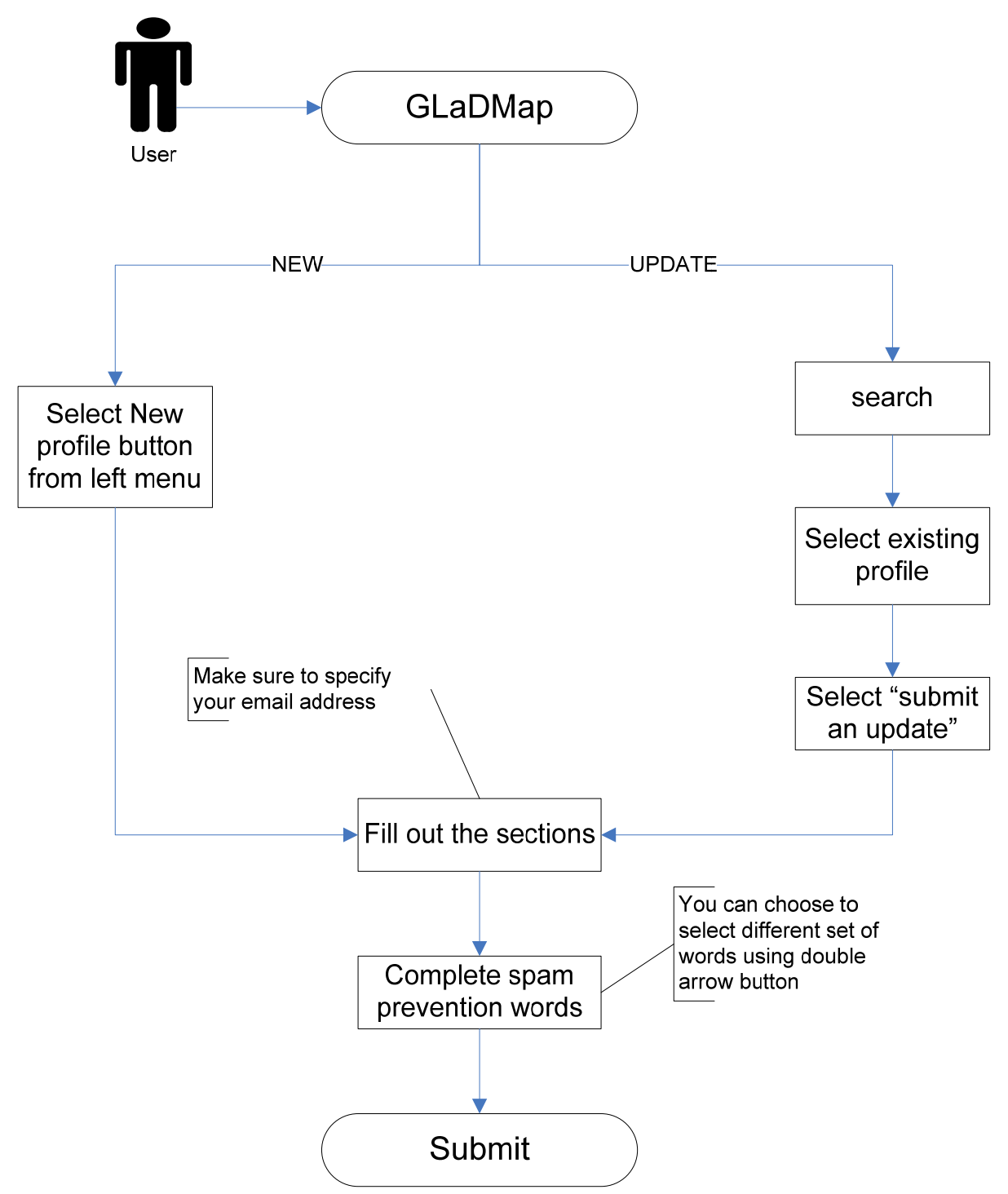

Fig. 4. GLaDMap: Process for submitting new profile and updates

\subsection{GLaDResource}

GLaDResource component of the GLaD platform is intended to be a dynamic, shared repository for archiving templates, tools/"best use" advice, and self-learning materials. It is intended to provide access to: 1) templates (agreements, contracts, multi-center study formats, etc.), 2) network tools (i.e. lexicon, managing membership, quality standard monitoring, etc.), protocols (for evaluations, reference panels, wet-laboratory exercises etc.) 
and successful solutions and techniques (advocacy techniques, shipping specimens, etc.), and 3) a self-learning e-platform offering topics for professionals so they can learn about scientific advancements, management practices and organizational skills.

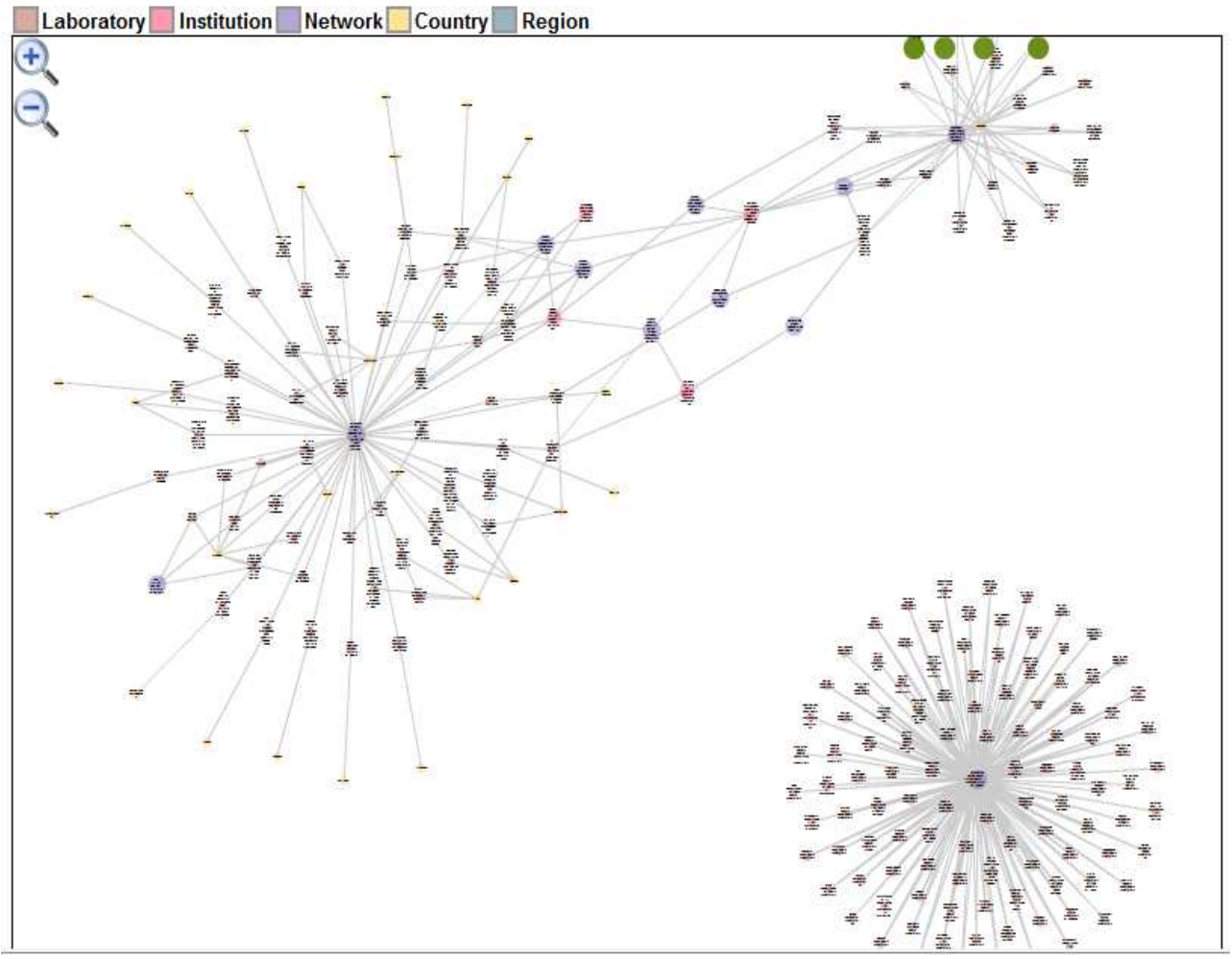

Fig. 5. Interactive Relationship Diagram: Illustration of multidimensional relationships

GLaDResource will be a secure site available to laboratory professionals. Resource sharing will be based on two concepts. First, participants would be able to upload video tutorials, PDF files, images or web links to a secure parking lot. The parking lot will enable users to ask questions and leave comments on the various resources within the lot, thus fostering rapid collaboration and assessment of resources on a global scale. Second, a system will be developed to automatically identify resources on the web using automated search engines to provide faceted searching, full-text searching, dynamic clustering and geospatial searching. Such a system will enable rapid identification of resources available on the Internet (such as, YouTube, e-journals,) and create an instant pool of such resources for the users.

Some of the examples of resources that will be considered for inclusion within the GLaDResource components include:

- Self-learning material to see how tests are done in various countries

- Quality standards for tests that are set by accreditation bodies (ISO, CAP, CLIA) 
- How various laboratories work within these systems, and how they differ based on tests, laboratory, network, country

- Use of proficiency panels and biosafety around the globe

- Protocols from highly regarded laboratories such as WHO reference centers

The following key concepts will be considered during the development of the GLaDResource component, keeping in mind that it needs to be user-driven system that empowers and encourages users to input resources and maintain them:

- Resources should be targeted to scientists, technicians laboratory directors, and network managers

- Format of files to make sure they are sharable, printable, et cetera

- Language of resources to make sure they can be translated with ease

- Quality of resources:

- Where do the protocols originate?

- What is the "status" of the author?

- Legal considerations

It is well understood that the willingness of users to collaborate and share documents will require the generation of immediate value-added to the community.

\subsection{GLaDSupport}

GLaDSupport is planned to be an activities platform linking networks and their members to interact within their network and with others using "closed" social networking platform. GLaDSupport will: 1) keep a message board for networks to post announcements, open invitations to meetings and training opportunities, 2) give strategic guidance and assistance to existing and new networks, 3) create opportunities to connect laboratory network managers and, 4) organize meetings and forums for sharing practical information, and proven best practices that keep networks functional and active.

A Social Network is a set of Processes, Methods and Information, Communication Technology (ICT) to bring communities and likeminded people together to interact for common purposes or serving common goals through provision of a platform for participants to socially interact with each other. Twitter, Facebook and LinkedIn are young (less than 10 year old) companies which have already proven the benefits of virtual social networking - reports of high profile events first appear on Twitter and Facebook, before the media network catches on. The individuals engaged in these social networks have a sense of belonging to the virtual society, and are interested in notifying their "social circle" about breaking events.

According to a recent article in the Wall Street Journal (Vascellero, 2007), social networking opportunities for professionals have been burgeoning with the recent advent of websites such as Sermo.com for physicians, AdGabber.com for advertising, and InMobile.org for the wireless industry. These websites are intended as a place for individuals in the same industry to connect, network, and share ideas. These websites differ from message boards in that they include features such as personal blogs, profiles with professional credentials, links to friends, instant messaging, and event invitations.

Online communities of practice can allow users to interact with others in their field without geographic or temporal barriers. This anywhere/anytime communication increases access to information from others in their field, and facilitates professional growth and knowledge 
transfer. According to (Sherer et. al., 2003), asynchronous Internet communication can supplement a community of practise by providing the following services:

- Question and answer: Such as a message board

- White pages: A directory of members

- $\quad$ Subject matter experts: A topical directory of experts

- Professional Development activities: informal, self- directed learning or a listing of face to face or online professional development programs

- Library: Resources to support the community focus

- Best practices: What has been done and what works well, including templates and examples

- Lessons learned: History and documentation of the community's progress

- Community management tools: following participants, traffic, most popular documents and discussions

- Community workspace: Collaborative tools for discussion (chat, discussion board, white board, videoconferencing) and ability of members to create their own special interest groups

- Site search engine: For ease of access to documents

- Other related communities: Links to other communities and websites

Because these communities may include a large amount of information pertaining to different topics, (Sherer et. al., 2003) also recommend allowing users to customize their experience so that they only see the information most relevant to themselves.

In designing an interactive community for professionals, it is necessary to understand how the community will be used and what type of knowledge will be shared. A study conducted in 2007 (Hara \& Hew, 2007) investigated why and how nurses used an online community of practise. The study found that nurses' use of the online community arose from a need to ask questions and interact with other nurses that are doing the same job that they do. Because it is necessary for nurses to be knowledgeable about current best practise methods and literature, they found the information available in the community useful. Many well-known nurses were members of the community, and this gave the community a level of expertise and professionalism that attracted more members. In terms of content of the community, researchers found that $51 \%$ of the messages regarded knowledge sharing, 33\% solicitation of help, and the remainder pertained to appreciation, job posting, empathy, greetings, administration, clarification, compliments, and encouragement. Furthermore, of the $51 \%$ of messages involving knowledge sharing, only $8.7 \%$ contained book knowledge being shared, while the rest contained practical knowledge. This shows that by far, participants were sharing practical knowledge acquired through practice in order to reduce gaps advance their skills as practitioners. The authors conclude that in order to sustain knowledge sharing in an online community, you need six factors:

1. Self- selected members: To ensure knowledge sharing is informal, voluntary, and natural

2. Validation of practise: To establish identity and reinforce role of practitioners

3. A need for knowledge: A need to be knowledgeable about current best practises, technology, and advancements in the field 
4. A non-competitive environment: Sharing knowledge with peers from different organization reduces competition and increases willingness to share, as there is no individual recognition involved

5. Asynchronous communication: Convenient, anytime- anywhere access

6. A moderator: To ensure ease of use by members and to keep the discussion focused and professional

Finally, in a global community, it is important to understand any cultural barriers to access. In a 2006 study done by the engineering firm Caterpillar (Ardichvilli et. al., 2006) in it's international offices, located in Brazil, China, and Russia, researchers investigated what factors might prevent employees from participating in an online community of practise with their international affiliates. They found that the most commonly cited barriers were:

- Modesty; fear of looking boastful or immodest

- Language barriers; concern that their English was not good enough to communicate with international colleagues

- Competitiveness and job security; the notion that knowledge is the key to employability, and by sharing that knowledge they lose their competitive edge

- Hierarchy; higher level employees may not find the time or may not see value in participation

- Preference for face-to-face communication over online communication

- Reluctance to share knowledge with members of "other" groups

The GLaDSupport component will utilize some of the above-mentioned principles and lessons learned in developing a novel closed social network for laboratory professionals to facilitate global collaboration and consultation.

\subsection{Scenarios}

The GLaD platform can provide significant benefits to the laboratory community. The following lists some example scenarios on how GLaD contributes to global collaboration and consultation while providing efficient means of finding relevant information:

- Toxicology Testing for Melamine: Traditionally, responsible organization would spend significant amount of time looking for information on laboratories that could perform melamine testing, typically through word of mouth. GLaD's search engine, if populated with such laboratory profiles ahead of time, can interactively and rapidly locate all toxicology laboratories and their locations, and provide the means to further narrow the search for melamine testing.

- Influenza Diagnostic Capability and Capacity: A laboratory network spends great effort and resources assessing Influenza capacity within its member laboratories, information, which becomes quickly out of date. GLaD provides a mechanism for laboratory profiles to be available online and kept up to date. Diagnostic gaps can be quickly addressed and equipment supplied without costly surveys.

Typical current practice is to search the Internet for information, which can be extremely time consuming and oftentimes misleading, or to use traditional approach of using phone calls to search for expertise. GLaD platform can provide a one-stop-shop for laboratory 
related information, which is fast, continually evolving, and universally accessible with a multi-faceted approach to searching and sharing.

\section{Conclusion}

Global health security is everyone's responsibility. Uniting scientists and their expertise into networks is one way to strengthen response, build capacity and share with others at local, regional and global levels. Successful models of cooperative networks-- Global Outbreak and Response Network, the UN agencies (WHO, Food and Agriculture Organization)supported networks, the World Animal Health Organization (OIE) reference networks, PulseNet International and its sub-regional networks-- are examples of functional networks and a testament that networks do provide the connection and are irreplaceable repositories of experience and knowledge. Paradoxically, even as the scientific community recognizes that it is easy to form a network, it also recognizes that sustaining the work of the networks can often be challenging. The development of knowledge management tools for this project is intended to assist the GLaD laboratory community to meet their challenges.

\section{Acknowledgment}

The authors would like to convey sincere thanks to Ms. Kashmeera Meghnath who performed literature review and usage analysis and assisted with editing of this chapter. The authors are grateful to the World Health Organization for supporting developing the GLaD conceptual ideas under the International Health Regulations Coordination Office, Geneva, Switzerland and to the laboratory technical advisers of their experience in managing global laboratory networks.

\section{References}

Ardichvilli, A, Maurer, M, Li, W, and Wentling, T. (2006). Cultural influences on knowledge sharing through online communities of practise. Journal of Knowledge Management. 10, 94-107.

Baker, M.G. \& Fidler, D.P. (2006). Global public health surveillance under new international health regulations. Emerging Infectious Diseases. 12:1058-65.

Featherstone, D., Brown, D., \& Sanders, R. (2003). Development of the global measles laboratory network. J Infect Dis. 187(Suppl 1):S264-9

Hara, N and Hew, KF. (2007). Knowledge sharing in an online community of health professionals. Information Technology \& People. 20, 235- 261.

Hull, B.P. \& Dowdle, W.R. (1997). Poliovirus surveillance: building the poliovirus laboratory network. J Infect Dis. 175(Suppl):S113-6

Mukhi, S. N., Aramini J. and Kabani A. (2007). Contributing to communicable disease intelligence management in Canada, Can J Infect Dis Med Microbiol. November; 18(6): 353-356.

Sherer, PD, Shea, TP, and Kristensen, E. (2003). Online Communities of Practice: A Catalyst for Faculty Development. Innovative Higher Education. 27, 183- 294. 
Vascellero, J.E. (2007). Social Networking Goes Professional. Wall Street Journal. Retrieved from http:/ / online.wsj.com/article/SB118825239984310205.html

World Health Organization (2008). International Health Regulations, 2005. ISBN 9789241580410. Accessed on September 03, 2011. Available from:

http://www.who.int/ihr/9789241596664/en/index.html 


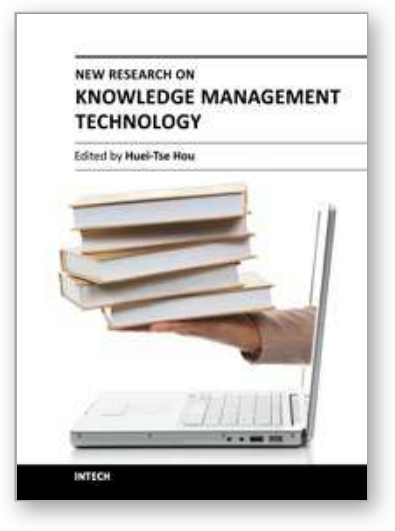

\author{
New Research on Knowledge Management Technology \\ Edited by Dr. Huei Tse Hou
}

ISBN 978-953-51-0074-4

Hard cover, 228 pages

Publisher InTech

Published online 24, February, 2012

Published in print edition February, 2012

Due to the development of mobile and Web 2.0 technology, knowledge transfer, storage and retrieval have become much more rapid. In recent years, there have been more and more new and interesting findings in the research field of knowledge management. This book aims to introduce readers to the recent research topics, it is titled "New Research on Knowledge Management Technology" and includes 13 chapters. In this book, new $\mathrm{KM}$ technologies and systems are proposed, the applications and potential of all KM technologies are explored and discussed. It is expected that this book provides relevant information about new research trends in comprehensive and novel knowledge management studies, and that it serves as an important resource for researchers, teachers and students, and for the development of practices in the knowledge management field.

\title{
How to reference
}

In order to correctly reference this scholarly work, feel free to copy and paste the following:

Shamir N. Mukhi, Lai King Ng, Theodore I. Kuschak and May Chu (2012). Knowledge Integration to Support Networking for Laboratory Preparedness and Response to Emerging Pathogens, New Research on Knowledge Management Technology, Dr. Huei Tse Hou (Ed.), ISBN: 978-953-51-0074-4, InTech, Available from: http://www.intechopen.com/books/new-research-on-knowledge-management-technology/knowledgeintegration-to-support-networking-for-laboratory-preparedness-and-response-to-emerging-pat

\section{INTECH}

open science | open minds

\author{
InTech Europe \\ University Campus STeP Ri \\ Slavka Krautzeka 83/A \\ 51000 Rijeka, Croatia \\ Phone: +385 (51) 770447 \\ Fax: +385 (51) 686166 \\ www.intechopen.com
}

\author{
InTech China \\ Unit 405, Office Block, Hotel Equatorial Shanghai \\ No.65, Yan An Road (West), Shanghai, 200040, China \\ 中国上海市延安西路65号上海国际贵都大饭店办公楼 405 单元 \\ Phone: +86-21-62489820 \\ Fax: +86-21-62489821
}


(C) 2012 The Author(s). Licensee IntechOpen. This is an open access article distributed under the terms of the Creative Commons Attribution 3.0 License, which permits unrestricted use, distribution, and reproduction in any medium, provided the original work is properly cited. 